\title{
Application of OLGA (Operative link on gastritis assessment) staging system for gastritis in alcoholics- a cross sectional study
}

\author{
Rakshitha HB ${ }^{1}$, Nandini GV ${ }^{2, *}$, Mangala Gouri ${ }^{3}$, Avinash Balekuduru ${ }^{4}$ \\ ${ }^{\mathbf{1}}$ Assistant Professor, Dept. of Pathology, Adichunchanagiri Institute of Medical Sciences, Karnataka, ${ }^{2}$ Assistant Professor, Dept. \\ of Pathology, Shridevi Institute of Medical Sciences and Research Hospital, Karnataka, ${ }^{3}$ Professor, ${ }^{4}$ Associate Professor, ${ }^{3}$ Dept. \\ of Pathology, ${ }^{4}$ Dept. of Gastroenterology, Ramaiah Medical College, Bangalore, Karnataka, India
}

\section{*Corresponding Author:}

Email: gvnandini@gmail.com

\begin{abstract}
Introduction: Gastritis is an inflammatory condition of the gastric mucosa. Atrophy of the gastric mucosa is the endpoint of chronic processes, such as chronic gastritis associated with $\mathrm{H}$. pylori infection.

Currently, no reporting scheme/terminology for chronic gastritis is available that is both easily understood by clinicians and patients and also provides prognostic and therapeutic information in unequivocal terms.

An international group Operative Link on Gastritis Assessment (OLGA) proposed a staging system for reporting gastric histology. This gastritis staging integrates the atrophy score (obtained by biopsy) and the atrophy topography (achieved through directed biopsy mapping).

This prospective cross-sectional study aimed to apply the OLGA staging system for reporting gastric histology in alcoholic patients with gastritis, thus validate the OLGA gastritis staging system as a routine histology reporting system.

Materials and Methods: A prospective study was conducted from October 2013 to June 2015 in the Department of Gastroenterology, M.S. Ramaiah Hospitals (M.S. Ramaiah Teaching Hospital and M.S. Ramaiah Memorial Hospital), Bangalore. 150 alcoholic patients were involved in the study. Age, sex, presenting complaints and detailed history regarding alcohol intake was taken followed by investigations like Complete Blood Count, Liver Function Tests, Renal Function Tests and ultrasound abdomen. Patients were categorised clinically into 4 groups as Alcohol Dependence Syndrome (ADS), fatty liver, Compensated Liver Disease (CLD) and Decompensated Liver Disease (DCLD). Rapid urease test was done for all the patients and 5 biopsy samples ( 2 from antral mucosa, 2 from oxyntic area and one from the mucosa of the incisura angularis.) were obtained and sent to the pathology department. Sections made from these gastric biopsies were stained with H and E and Giemsa stain. Sections were then evaluated for the presence of $\mathrm{H}$. pylori. Severity of atrophic gastritis was noted and finally OLGA scoring was given for each patient.

Results: In the 150 alcoholic patients included in the study, prevalence of H. pylori was $76 \%$. The age and the gender did not play a role in the H. pylori infection.

ADS constituted 53 patients (35.3\%), Fatty liver 21 (14\%), CLD 39 (26\%), DCLD 37 (24.7\%). H. pylori prevalence was 60.4\%, $71.4 \%, 82.1 \%$ and $94.6 \%$ respectively.

With the increase in OLGA staging, number of $\mathrm{H}$. pylori positive patients increased, which is statistically significant (p value is $<0.001)$.

Majority of the patients with ADS belonged to OLGA stage 0 (37 cases, 69.8\%) and majority of the patients with DCLD belonged to OLGA stage II ( 26 cases, $70.3 \%$ ). The current study revealed that the OLGA staging increased with worsening of the ALD.

Conclusion: The OLGA staging increases as the clinical presentation of ALD worsens. With the increase in OLGA staging, number of $\mathrm{H}$. pylori positive patients increased. The new OLGA staging system proved easy to use and provides clear prognostic information.
\end{abstract}

Keywords: H. pylori, OLGA, Alcohol intake, ALD.

\section{Introduction}

Gastritis is an inflammatory condition of the gastric mucosa with different aetiologies, phenotypic expressions and natural histories. ${ }^{1,2}$ Longstanding inflammation of the gastric mucosa is thought to trigger a cascade of genotypic and phenotypic derangements that may eventually result in intestinal-type (or epidemic) gastric cancer (GC). ${ }^{3,4}$ Gastric mucosal atrophy is the soil in which GC most frequently develops and different patterns of atrophic- metaplastic gastritis are associated with different GC risks. ${ }^{4-9}$ Atrophy of the gastric mucosa is the endpoint of chronic processes, such as chronic gastritis associated with H. pylori infection, other unidentified environmental factors, and autoimmunity directed against gastric glandular cells. Atrophy of the gastric mucosa (with and without intestinal metaplasia) is considered the "field cancerization" for the development of gastric cancer. ${ }^{10-14}$

Currently, the available classifications of gastritis are inconsistently used possibly because none provides immediate prognostic/therapeutic information to clinicians.

So an international group Operative Link on Gastritis Assessment (OLGA) $)^{15}$ proposed an equivalent staging system for reporting gastric histology. This gastritis staging integrates the atrophy score (obtained 
by biopsy) and the atrophy topography (achieved through directed biopsy mapping).

This prospective cross-sectional study aimed to apply the OLGA staging system for reporting gastric histology in alcoholic patients with gastritis, thus validate the OLGA gastritis staging system as a routine histology reporting system.

\section{Materials and Methods}

Source of Data: Gastric biopsies from alcoholic patients received for routine histopathological evaluation from the Department of Gastroenterology, M.S. Ramaiah Hospitals (M.S. Ramaiah Teaching Hospital and M.S. Ramaiah Memorial Hospital), Bangalore, from October 2013 to June 2015 were the source of data for the study.

Inclusion Criteria: Patients with history of alcohol intake presenting with upper gastrointestinal symptoms to M.S. Ramaiah Hospitals to Medicine/Gastroenterology Services and consenting for gastroduodenoscopy and biopsy were included in the study.

\section{Exclusion Criteria:}

1. Patients who are not consenting for endoscopy.

2. Patients who are on anti-biotics.

3. Inadequate samples.

Method of Collection of Data: Age, sex and presenting complaints of the patients were noted. A detailed history was collected from each patient regarding alcohol intake.

Following investigations were done for all the patients- Complete blood count, Liver function tests (LFT), coagulation profile, Renal Function Tests (RFT) and Ultrasound abdomen. Then, all the 150 patients were categorised clinically into four different categories as
1. Alcohol dependence syndrome (ADS)

2. Fatty liver (FL)

3. Compensated liver disease (CLD)

4. Decompensated liver disease (DCLD)

Alcohol Dependence Syndrome (ADS) was categorised based on CAGE questionnaire and Liver Function Tests:

CAGE questionnaire, the name of which is an acronym of its four questions, is a widely used screening test for alcoholism. ${ }^{16}$

Two "yes" responses indicate that the patient belongs to ADS category.

The questionnaire asks the following questions:

1. Have you ever felt you needed to cut down on your drinking?

2. Have people annoyed you by criticizing your drinking?

3. Have you ever felt Guilty about drinking?

4. Have you ever felt you needed a drink first thing in the morning (Eye-opener) to steady your nerves or to get rid of a hangover?

The CAGE questionnaire is validated for use in identifying alcoholism.

Fatty liver was Categorised Based on: The presence of fatty infiltrates in the liver detected by ultrasound scan of the abdomen and deranged LFT.

CLD was Categorised Based on: History of alcohol intake, deranged LFT and coagulation profile, endoscopic and ultrasound findings of varices.

DCLD was Categorised Based on: History, clinical findings and investigation reports with similar findings as in CLD but with the presence of additional complications of liver cirrhosis like portal hypertension, hepatic encephalopathy, ascites and oesophageal varices. The categorisation of CLD and DCLD was mainly based on Child-Pugh score. ${ }^{16}$

\begin{tabular}{lccc}
\hline Child-Pugh score & \multicolumn{3}{c}{ Scores } \\
\cline { 2 - 4 } Clinical and laboratory & $\mathbf{1}$ & $\mathbf{2}$ & $\mathbf{3}$ \\
parameter & None & $1-2$ & $3-4$ \\
Encephalopathy (grade) & None & Slight & Moderate \\
Ascites & $>3.5$ & $2.8-3.5$ & $<2.8$ \\
Albumin (g/dL) & $1-4$ & $4-6$ & 6 \\
Prothrombin time prolonged (s) & $<2$ & $2-3$ & $>3$ \\
Bilirubin (mg/dL) & $<4$ & $4-10$ & $>10$ \\
For primary biliary cirrhosis & \multicolumn{4}{c}{ Class A=5-6 points; Class B=7-9 points; Class C=10-15 points. Class A: Good } \\
operative risk. Class B: Moderate operative risk. Class C: Poor operative risk \\
\hline
\end{tabular}

\section{Fig. 1: Child-pugh score}

Patients under class A were categorised as CLD and patients under class $\mathrm{B}$ and $\mathrm{C}$ were categorised as DCLD.

Written consent was taken from each patient and endoscopy was performed by the gastroenterologist and 5 gastric biopsy samples were collected from each patient, 2 from antral mucosa, 2 from oxyntic area and one from the mucosa of the incisura angularis. Apart from these 5 biopsies, an additional biopsy sample was collected from each patient from the antrum, for Rapid Urease Test (RUT). Commercially available Pylo Dry kit was used for the RUT test. 
The change in the colour of the Pylo Dry kit media from yellow to pink was taken as a positive test.

The 5 biopsy samples obtained were fixed in formalin $(10 \%)$ and submitted to the department of pathology in three separate vials, labelled according to their topographic site (antrum, incisura angularis, and corpus). The paraffin-embedding procedure distinguished the biopsy samples accordingly.

Two histology sections (5 micrometers thick) were obtained from each paraffin block. Sections were stained with H\&E and Giemsa for all the cases.

Microscopic examinations of the sections were carried out. Microscopically, in each case, presence of H. pylori was looked for, both in $\mathrm{H}$ and $\mathrm{E}$ stained sections (Fig. 3) and Giemsa stained sections (Fig. 4).

Also at each biopsy sample site, gastritis was distinguished as non-atrophic (i.e. no loss of appropriate glands) and atrophic (i.e. loss of appropriate glands).

Mucosal atrophy was scored according to the Sydney system using a visual analogue scale as follows: For each patient, the three biopsy samples, two obtained from the antrum and one sample obtained from incisura angularis were considered together, as representative of the distal gastric mucosa and the average percentage of atrophy was obtained from these three samples. Then finally, the atrophy score for antrum was obtained from the calculated average percentage of atrophy (if no atrophy then score $=0$; if atrophy is between $1-30 \%$ it is mild atrophy, score $=1$; if atrophy is between $30-60 \%$ it is moderate atrophy, score $=2$; if atrophy is $>60 \%$ then it is severe atrophy, score $=3$ ).

Likewise the two biopsy samples obtained from the corpus were considered together, as representative of proximal gastric mucosa, and the average percentage of atrophy for both the samples were calculated. Then the atrophy score for corpus was obtained from the calculated average percentage of atrophy. If no atrophy then score $=0$; if atrophy is between $1-30 \%$ it is mild atrophy, score $=1$; if atrophy is between $30-60 \%$ it is moderate atrophy, score $=2$; if atrophy is $>60 \%$ then it is severe atrophy, score=3. (Fig. 5-7)

The OLGA gastritis stage was finally obtained by combining the atrophy score of antrum with the atrophy score of corpus (Fig. 2)

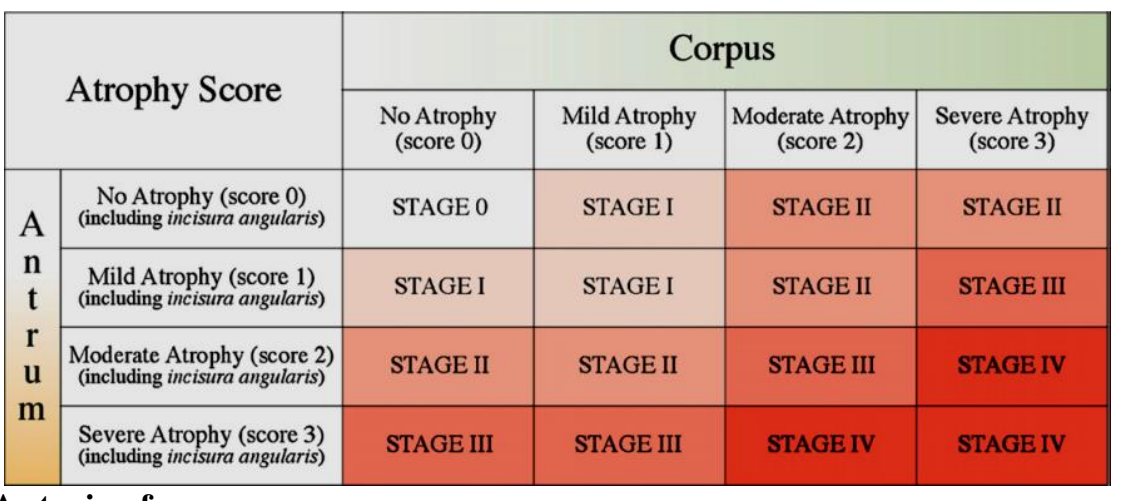

\section{Fig. 2: The OLGA staging frame}

Finally the OLGA staging obtained was correlated with the $\mathrm{H}$. pylori status and the clinical presentation of the patients.

Statistical analysis: Descriptive statistics of $\mathrm{H}$. pylori has been analysed and presented in terms of percentage and its $95 \%$ confidence interval is estimated. Chi-
Square test is used to compare the H. pylori proportion between age, sex, type, frequency and amount of alcohol intake.

\section{Results}

The results obtained are as follows:

Table 1: Age and sex distribution

\begin{tabular}{|l|c|c|c|}
\hline \multirow{2}{*}{ Age } & \multicolumn{2}{|c|}{ Sex } & \multirow{2}{*}{ Total } \\
\cline { 2 - 4 } & Male & Female & 48 \\
\hline \multirow{2}{*}{$21-40$} & 48 & 0 & $32.0 \%$ \\
\cline { 2 - 4 } & $32.9 \%$ & $0.0 \%$ & 93 \\
\hline \multirow{2}{*}{$61-60$} & 92 & 1 & $62.0 \%$ \\
\cline { 2 - 4 } & $63.0 \%$ & $25.0 \%$ & 9 \\
\hline \multirow{2}{*}{ Total } & 6 & 3 & $6.0 \%$ \\
\cline { 2 - 4 } & $4.1 \%$ & $75.0 \%$ & 150 \\
\hline
\end{tabular}


Of the 150 cases, maximum number of patients were in the age group of 41-60 years (62.0\%) and only $6 \%$ of the cases were between 61-80 years of age.

Of the 150 patients, 146 were males and 4 were females.

Table 2: Gender distribution in the study

\begin{tabular}{|l|c|c|}
\hline & Number & Percent \\
\hline Female & 4 & 2.7 \\
\hline Male & 146 & 97.3 \\
\hline Total & 150 & 100.0 \\
\hline
\end{tabular}

Table 3: H. pylori positivity by RUT

\begin{tabular}{|l|c|c|}
\hline \multicolumn{1}{|c|}{ RUT } & Number & Percent \\
\hline Negative & 36 & 24.0 \\
\hline Positive & 114 & 76.0 \\
\hline Total & 150 & 100.0 \\
\hline
\end{tabular}

Table 4: H. pylori positivity in biopsy

\begin{tabular}{|l|c|c|}
\hline & Number & Percent \\
\hline $\begin{array}{l}\text { Chronic non } \\
\text { specific gastritis }\end{array}$ & 36 & 24.0 \\
\hline $\begin{array}{l}\text { H pylori induced } \\
\text { chronic gastritis }\end{array}$ & 114 & 76.0 \\
\hline Total & 150 & 100.0 \\
\hline
\end{tabular}

Table 5: Categorizing OLGA staging

\begin{tabular}{|l|c|c|}
\hline \multicolumn{1}{|c|}{$\begin{array}{c}\text { OLGA } \\
\text { staging }\end{array}$} & $\begin{array}{c}\text { Number of } \\
\text { patients }\end{array}$ & Percent \\
\hline Stage 0 & 49 & 32.7 \\
\hline Stage I & 50 & 33.3 \\
\hline Stage II & 47 & 31.3 \\
\hline Stage III & 4 & 2.7 \\
\hline Total & 150 & 100.0 \\
\hline
\end{tabular}

Of the 150 patients, 49 were categorized as OLGA stage 0,50 patients belonged to OLGA stage I, 47 patients belonged to stage II and 4 patients belonged to stage III.

Table 6: Presenting complaints

\begin{tabular}{|l|c|c|}
\hline $\begin{array}{c}\text { Presenting } \\
\text { complaints }\end{array}$ & Number & Percent \\
\hline Distention & 25 & 16.7 \\
\hline Malena & 8 & 5.3 \\
\hline Dyspagia & 1 & 0.7 \\
\hline Jaundice & 7 & 4.7 \\
\hline Pain abdomen & 77 & 51.3 \\
\hline Vomiting & 32 & 21.3 \\
\hline Total & 150 & 100.0 \\
\hline
\end{tabular}

Most common presenting complaint in the study population was pain abdomen, followed by vomiting.

Table 7: Clinical category and OLGA staging

\begin{tabular}{|c|c|c|c|c|c|}
\hline \multirow{2}{*}{ Clinical category } & \multicolumn{4}{|c|}{ OLGA Stage } & \multirow[t]{2}{*}{ Total } \\
\hline & Stage 0 & Stage I & Stage II & Stage III & \\
\hline \multirow{2}{*}{ Alcohol dependence syndrome } & 37 & 15 & 1 & 0 & 53 \\
\hline & $69.8 \%$ & $28.3 \%$ & $1.9 \%$ & $0.0 \%$ & $100.0 \%$ \\
\hline \multirow[t]{2}{*}{ Fatty liver } & 9 & 11 & 1 & 0 & 21 \\
\hline & $42.9 \%$ & $52.4 \%$ & $4.8 \%$ & $0.0 \%$ & $100.0 \%$ \\
\hline \multirow[t]{2}{*}{ Chronic liver disease } & 3 & 17 & 19 & 0 & 39 \\
\hline & $7.7 \%$ & $43.6 \%$ & $48.7 \%$ & $0.0 \%$ & $100.0 \%$ \\
\hline \multirow[t]{2}{*}{ Decompensated liver disease } & 0 & 7 & 26 & 4 & 37 \\
\hline & $0.0 \%$ & $18.9 \%$ & $70.3 \%$ & $10.8 \%$ & $100.0 \%$ \\
\hline \multirow[t]{2}{*}{ Total } & 49 & 50 & 47 & 4 & 150 \\
\hline & $32.7 \%$ & $33.3 \%$ & $31.3 \%$ & $2.7 \%$ & $100.0 \%$ \\
\hline
\end{tabular}

Chi square value 102.091, degrees of freedom- 9 , $p$ value is $<0.001$; Kappa value- 0.298

Of the 150 patients, clinically 53 patients belonged to the category of alcohol dependence syndrome, 21 were in fatty liver category, 39 patients in chronic liver disease category and 37 patients in decompensated liver disease category. Majority of the patients with alcohol dependence syndrome belonged to OLGA stage 0 (37 cases, 69.8\%). Majority of the patients with decompensated liver disease belonged to OLGA stage II ( 26 cases, $70.3 \%)$

Table 8: OLGA staging and H. pylori infection

\begin{tabular}{|l|c|c|c|}
\hline \multirow{2}{*}{ OLGA staging } & \multicolumn{2}{|c|}{ H. pylori infection } & \multirow{2}{*}{ Total } \\
\cline { 2 - 4 } & Positive & Negative & \\
\hline \multirow{2}{*}{ Stage 0} & 22 & 27 & 49 \\
\cline { 2 - 4 } & $44.9 \%$ & $55.1 \%$ & $100.0 \%$ \\
\hline \multirow{2}{*}{ Stage I } & 44 & 6 & 50 \\
\cline { 2 - 4 } & $88.0 \%$ & $12.0 \%$ & $100.0 \%$ \\
\hline
\end{tabular}




\begin{tabular}{|l|c|c|c|}
\hline \multirow{2}{*}{ Stage II } & 45 & 2 & 47 \\
\cline { 2 - 4 } & $95.7 \%$ & $4.3 \%$ & $100.0 \%$ \\
\hline \multirow{2}{*}{ Stage III } & 3 & 1 & 4 \\
\cline { 2 - 4 } & $75.0 \%$ & $25.0 \%$ & $100.0 \%$ \\
\cline { 2 - 4 } Total & 114 & 36 & 150 \\
\hline
\end{tabular}

Chi- square 39.112, degrees of freedom-2, $\mathrm{p}$ value is $<0.001$

Of the 150 patients, 49 patients belonged to OLGA stage 0 , of which $22(44.9 \%)$ patients were $\mathrm{H}$. pylori positive. 50 patients were categorised as OLGA stage I, of which $44(88.0 \%)$ patients were H. pylori positive. 47 patients were categorized as OLGA stage II, of which $45(95.7 \%)$ patients were $\mathrm{H}$. pylori positive. 4 patients were categorised as OLGA stage III, of which $3(75.0 \%)$ patients were $H$. pylori positive. With the increase in the OLGA staging, number of $\mathrm{H}$. pylori positive patients also increased, which is statistically significant.

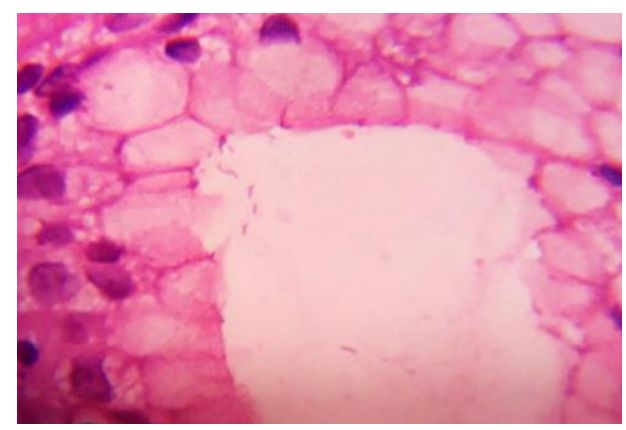

Fig. 3: H. Pylori (H and E stained section)

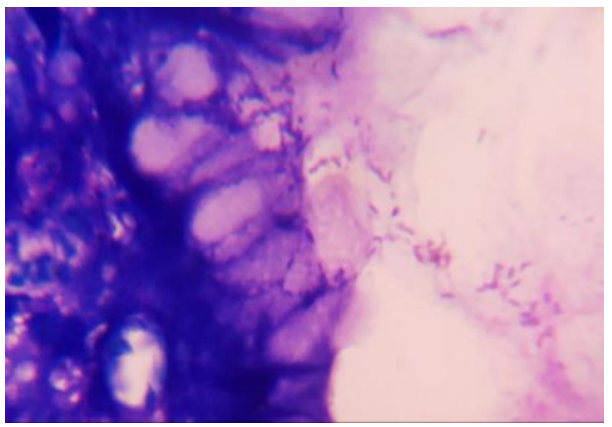

Fig. 4: H. pylori (Giemsa stained section)

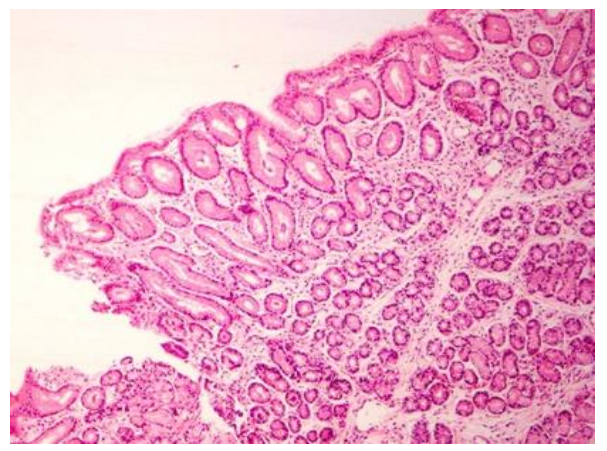

Fig. 5: Mild gastric atrophy
Fig. 6: Moderate gastric atrophy
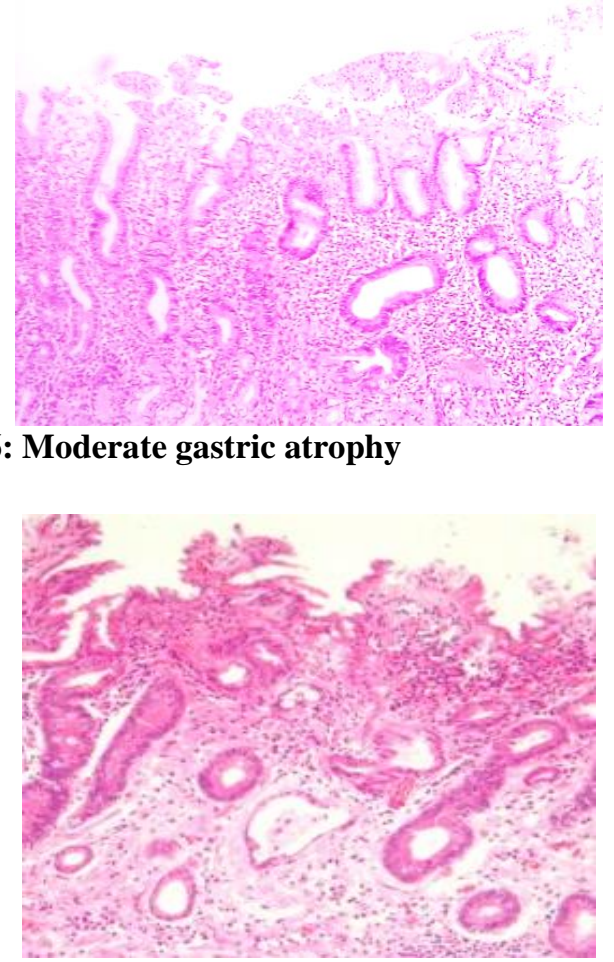

Fig. 7: Severe gastric atrophy

\section{Discussion}

150 patients were involved in the present study of which $114(76 \%)$ tested positive for H. pylori infection, by both RUT test and biopsy. According to this study the prevalence of $\mathrm{H}$. pylori in alcoholic individuals is $76 \%$.

Age and Sex Distribution in the Study Group: According to the current study, age and sex of the patient did not play a role in $\mathrm{H}$. pylori positivity and was similar to the results obtained in the previous studies by Li Zhang et al. ${ }^{17}$

OLGA Staging and Correlation with the Clinical Categories: Of the 150 patients, 49 patients belonged to OLGA stage 0 , of which 22 (44.9\%) patients were $\mathrm{H}$. pylori positive, 50 patients belonged to OLGA stage I, of which $44(88.0 \%)$ patients were H. pylori positive, 47 patients belonged to OLGA stage II, of which 45 (95.7\%) patients were $\mathrm{H}$. pylori positive and 4 patients belonged to OLGA stage III, of which 3 (75.0\%) were $\mathrm{H}$. pylori positive. With the increase in the OLGA staging, number of $\mathrm{H}$. pylori positive patients also increased, which is statistically significant.

The study population was divided into 4 groups and the prevalence of $\mathrm{H}$. pylori was studied in each group. ADS constituted 53 patients (35.3\%), Fatty liver 
21 (14\%), CLD 39 (26\%), DCLD 37 (24.7\%). H. pylori prevalence was $60.4 \%, 71.4 \%, 82.1 \%$ and $94.6 \%$ respectively. The frequency of $\mathrm{H}$. pylori was compared between the groups, prevalence of $\mathrm{H}$. pylori was the highest in the DCLD group as compared to the ADS group and this was statistically significant with a $p$ value of 0.002 . This study revealed that the $\mathrm{H}$. pylori prevalence increases with worsening of the ALD.

Tsai et al found that the prevalence of $\mathrm{H}$. pylori was similar in compensated and decompensated cirrhotic patients but Naumovski-Mihalie et al reported that $\mathrm{H}$. pylori infection was higher in decompensated patients which was similar to the results obtained in the present study. ${ }^{18,19}$

The OLGA staging obtained was compared with the clinical categories in the study group. It was observed that, majority of the patients with alcohol dependence syndrome belonged to OLGA stage 0 (37 cases, $69.8 \%$ ) and majority of the patients with decompensated liver disease belonged to OLGA stage II (26 cases, $70.3 \%)$. The current study also revealed that the OLGA staging increases with worsening of the ALD.

\section{Conclusion}

The OLGA staging increases as the clinical presentation of ALD worsens. With the increase in OLGA staging, number of $\mathrm{H}$. pylori positive patients increased. The new OLGA staging system proved easy to use and provides clear prognostic information.

The OLGA stage should be given as the concluding statement of a traditional histology report.

The OLGA staging information should be combined with the aetiological hypothesis of the gastric disease eg, $H p+v e, H p-v e, \quad$ or $H p$-suspected, or histology consistent with autoimmune gastritis) as suggested by the histological features (eg, OLGA staging: stage IV $(\mathrm{A}=3 ; \mathrm{C}=2) ; H p+v e)$.

\section{References}

1. Correa P. The epidemiology and pathogenesis of chronic gastritis: three etiologic entites. Journal of Gastrointestinal Research. 1980;6:98-108.

2. Graham DY. Helicobacter pylori infection in the pathogenesis of duodenal ulcer and gastric cancer: a model. Gastroenterology. 1997;113:1983-91.

3. Schistosomes, liver flukes and Helicobacter pylori. IARC Working Group on the Evaluation of Carcinogenic Risks to Humans 1994;61:1-241.

4. Uemura N, Okamoto S, Yamamoto S. Helicobacter pylori infection and the development of gastric cancer. New England Journal of Medicine. 2001;345:784-9.

5. Comfort MW. Gastric acidity before and after development of gastric cancer: its etiologic, diagnostic and prognostic significance. Annals of Internal Medicine. 1951;34:1331-48.

6. Filipe MI, Munoz N, Matko. Intestinal metaplasia types and the risk of gastric cancer: a cohort study in Slovenia. International Journal of Cancer. 1994;57:324-9.

7. Rugge M, Correa P, Dixon MF. Gastric mucosal atrophy: interobserver consistency using new criteria for classification and grading. Alimentary Pharmacology and Therapeutics. 2002;16:1249-59.

8. Rugge M. Secondary prevention of gastric cancer. Gastroenterology. 2007;56:1646-7.

9. Sung JJ, Kuipers EJ, El-Serag HB. Systematic review: the global incidence and prevalence of peptic ulcer disease. Alimentary Pharmacology and Therapeutics 2009; 29: 938-46.

10. Stemmermann G. Intestinal metaplasia of the stomach. A status report. Cancer. 1994;74(2):556-64.

11. Garcia S, Park HS, Novelli M. Field cancerization, clonality, and epithelial stem cells: the spread of mutated clones in epithelial sheets. Journal of Pathology. 1999;187(1):61-81.

12. Matsushima $\mathrm{K}$, Isomoto $\mathrm{H}$, Inoue $\mathrm{N}$, Nakayama $\mathrm{T}$, Hayashi T, Nakayama M, Nakao K, Hirayama T and Kohno S. MicroRNA signatures in Helicobacter pyloriinfected gastric mucosa. International Journal of Cancer. 2011;128:361-70.

13. Vannella L, Lahner E, Osborn J. Risk factors for progression to gastric neoplastic lesions in patients with atrophic gastritis. Alimentary Pharmacology and Therapeutics. 2010;31(9):1042-50.

14. Niwa T, Tsukamoto T, Toyoda T, et al. Inflammatory processes triggered by Helicobacter pylori infection cause aberrant DNA methylation in gastric epithelial cells. Cancer Research. 2010;70(4):1430-40.

15. Ruqqe M, Meqqio A, Pennelli G, Piscioli F, Giacomelli $\mathrm{L}$, De Pretis $\mathrm{G}$ et al. Gastritis staging in clinical practice: the OLGA staging system. Gastroenterology. 2007;56:631-6.

16. Harrison. Disorders of the gastrointestinal system. In:Harrison's principles of internal medicine, $19^{\text {th }}$ edition, volume 2, McGraw-Hill education, New York; 2012. 1989-2102.

17. Zhang, L, Guy D, Eslick, Harry H.-X.Xia, Chengqiu Wu, Nghi Phung, Nicholas J. Talley. Relationship between alcohol consumption and active Helicobacter pylori infection. Alcohol and Alcoholism. 2010;45(1):89-94.

18. Tsai C J. Helicobacter Pylori Infection and Peptic Ulcer Disease in Cirrhosis. Digestive Diseases and Science. 1998;43:1219-25.

19. Naumovski-Mihalie S, Colic - Cvrtje V, Prskato M, Saboric B, Tick M. Helicobacter Pylori Infection in Patients with Liver Cirrhosis. Gastroenterology. 2000;47:101-6.

How to cite this article: Rakshitha HB, Nandini GV, Gouri M, Balekuduru A. Application of OLGA (Operative link on gastritis assessment) staging system for gastritis in alcoholics- a cross sectional study. J Diagn Pathol Oncol. 2018;4(3):208-213. 